\title{
BCCN4
}

\section{DIFFERENT APPROCHES TO KINETICS DEGRADATION OF POLYESTER RESIN FROM RENEWABLE RESOURCES}

\author{
Vinicius Moraes Kieling da Rocha ${ }^{(1)}$, Heitor Luis Ornaghi Júnior ${ }^{(1)}$, Sandro Campos \\ Amico ${ }^{(1)}$, Daniel Magalhães de Oliveira ${ }^{(2)}$ and Kelly Cristina Coelho Benini (2) \\ (1) PPGE3M, Federal University of Rio Grande do Sul, Brazil \\ (2) Department of Materials and Technology, São Paulo State University (UNESP), Scholl of \\ Engineering, Guaratinguetá, São Paulo, Brazil (12156-410)
}

https://doi.org/10.21452/bccm4.2018.04.02

\begin{abstract}
Different approaches to the kinetic analysis of the thermal degradation of polyester resins, one traditional and its green alternative from renewable sources, were studied. The first step to determine the degradation mechanism was to find the following kinetic parameters: activation energy $\left(E_{a}\right)$ and the pre-exponential factor $(A)$. For the study it was used four distinct methods: the Flynn-Wall-Ozawa (FWO), the Friedman, the Kissinger and the modified Coats-Redfern methods. The second step was to find the most probable degradation mechanism based on the kinetic parameters obtained so far. In this study was used master plots proposed by Criado's. The mean activation energy values, named $E_{\text {ISo }}$, and the preexponential factor $(\ln (A))$ for the traditional polyester resin were $189.79 \pm 37.82 \mathrm{~kJ}$ and $24.62 \pm 6.11 \mathrm{~min}^{-1}$, respectively. For the resin from renewable resources, the values of $E_{a}$ and $\ln (A)$ were $161.85 \pm 14.35 \mathrm{~kJ}$ and $18.42 \pm 2.36 \mathrm{~min}^{-1}$, respectively. For both resins, the most probable degradation mechanism in all conversion range was nucleation and growth type mechanism. The methodology used in this study indicates that the kinetic parameters can be obtained in a reliable way, since combined.
\end{abstract}

\section{INTRODUCTION}

Concerts over the future availability of natural resources and the amount of produced waste have driven the need for the efficient use of these resources, aiming at sustainable development. The requirement for ecomaterials increased [1], and they are defined as materials manufactured from renewable resources and/or from the reuse of accumulated wastes in the environment, and that are also atoxic, among other characteristics [2].

Conventional thermoset polymers can be replaced by their green alternatives, as long as they maintain satisfactory properties. Thermal degradation, for instance, is an important characteristic for processing as well as in the definition of their applications. Its reliable prediction is an important tool to identify potential use of the material. 
In this present work, different approaches to the kinetic analysis of the thermal degradation of a traditional polyester resin and its green alternative from renewable resources were studied.

\section{TEORETICAL APPROACH}

\section{$2.1 \quad$ Degradation Kinetics}

Thermogravimetric analysis can be used to determine the degradation kinetics and to obtain reliable values of factor frequency, activation energy and general reaction order, i.e. the kinetic triplet [3]. Kinetic parameters were calculated according to ICTAC recommendations [4].

The main equation for kinetics is:

$\frac{d \alpha}{d t}=k(T) f(\alpha)$

where $k(T)=A \exp \left(-E_{a} / R T\right), f(\alpha)=(1-\alpha)^{n}, A$ is the pre-exponential factor, $E_{a}$ is the activation energy, $R$ is the gas constant $\left(8.314 \mathrm{~J} \mathrm{kmol}^{-1}\right), T$ is the absolute temperature and $n$ is the reaction order.

Including the $\beta$ term, i.e. heating rate, Equation (2) is obtained:

$$
\frac{d \alpha}{d t}=\frac{A}{\beta} e^{\left(-\frac{E_{a}}{R T}\right)} f(\alpha)
$$

The activation energy can be determined using distinct methods, including isoconversional Flynn-Wall-Ozawa (FWO), Friedman and Kissinger methods.

In the Kissinger method, the maximum of the reaction happens at the peak of the differential thermal analysis (DTA). This peak happens when the derivative of Equation (3) is zero. The activation energy for Kissinger can be determined plotting $\ln \left(\beta / T_{m}{ }^{2}\right)$ versus $1 / T_{m}^{2}$.

$\frac{d \alpha}{(1-\alpha)^{n}}=\left[\frac{A e^{\left(-\frac{E_{0}}{R T}\right)}}{\beta}\right] d T$

FWO is a conversional method, defined by Equation (4). Plotting $\log (\beta)$ versus $1 / T$ and applying a linear fit the factor frequency and the activation energy can be obtained. The slope gives $E_{a}$ and the intercept gives $A$.

$\log (\beta)=\log \left(A E_{a} / R g(\alpha)\right)-2.315-0.4567\left(E_{a} / R T\right)$

The Friedman method is defined by Equation (5). By plotting $\ln (d \alpha / d t)$ versus $1 / T$ and applying a linear fit the factor frequency and the activation energy can be obtained. The slope gives $E_{a}$ and the intercept gives $A$.

$\ln \left(\frac{d \alpha}{d t}\right)=\ln [A f(\alpha)]-\frac{E_{\alpha}}{R T}$

\subsection{Degradation Mechanism}

The activation energy of one solid-state reaction can be determined without knowing the mechanism of degradation. So, $E_{a}$ can be determined by isothermal or dynamics processes. After determination of the mean $E_{a}$ (by isothermal methods), the most probable degradation can be estimated by comparing master plots using the method proposed by Criado [5] Equation (6). 
$Z(\alpha)=\frac{(d \alpha / d t)}{\beta} \pi(X) T$

Peterson [6], proposed a relationship between $\pi(X)$ and the rotational equation $P(x)$, defined in Equation (7).

$\pi(x)=x e^{x} P(x)$

Senum and Yang [7], proposed a forth degree rational equation for $P(x)$. This was one of the equations used to estimate the precision of the Arrhenius integral and allowed a controlled margin of error. For the forth degree ration, the error is lower than $10^{-5} \%$ for $x>20$.

Combination of Equations (1), (6) and (7) yields, Equation (8). $\pi(x)=f(\alpha) g(\alpha)$

With Equation (8), it is possible to obtain theoretical thermogravimetric equations. The equations for $f(\alpha)$ and $g(\alpha)$ are determine according to the appropriate mechanism, as described in Table 1.

Table 1: The solid-state reaction mods and mechanisms of degradation.

\begin{tabular}{lcc}
\hline $\begin{array}{c}\text { Mechanism - Solid-state } \\
\text { reaction }\end{array}$ & $f(\alpha)-$ Reaction mode & $g(\alpha)-$ Reaction mode \\
\hline $\begin{array}{l}A_{2} \text { (Avrami-Erofeev) - } \\
\text { nucleation and growth }\end{array}$ & $2(1-\alpha)[-\ln (1-\alpha)]^{1 / 2}$ & {$[-\ln (1-\alpha)]^{1 / 2}$} \\
$\begin{array}{l}A_{3} \text { (Avrami-Erofeev) }- \\
\text { nucleation and growth }\end{array}$ & $3(1-\alpha)[-\ln (1-\alpha)]^{2 / 3}$ & {$[-\ln (1-\alpha)]^{1 / 3}$} \\
$\begin{array}{l}A_{4}(\text { Avrami-Erofeev) }- \\
\text { nucleation and growth }\end{array}$ & $4(1-\alpha)[-\ln (1-\alpha)]^{3 / 4}$ & {$[-\ln (1-\alpha)]^{1 / 4}$}
\end{tabular}

$R_{1}$ - Controlled reaction in the phase contour 1

(one-way movement)

$R_{2}$ - Controlled reaction in the phase contour (contracted area)

\section{1}

$\alpha$

$\begin{array}{cc}(1-\alpha)^{1 / 2} & 2\left[1-(1-\alpha)^{1 / 2}\right] \\ (1-\alpha)^{2 / 3} & 3\left[1-(1-\alpha)^{1 / 3}\right] \\ 1 / 2 \alpha^{-1} & 3\left[1-(1-\alpha)^{1 / 3}\right] \\ {[-\ln (1-\alpha)]^{-1}} & \alpha^{2} \\ (3 / 2)\left[1-(1-\alpha)^{1 / 3}\right]^{2}(1-\alpha)^{2 / 3} & (1-\alpha) \ln (1-\alpha)+\alpha \\ (3 / 2)\left[1-(1-\alpha)^{-1 / 3}\right]^{-1} & {\left[1-(1-\alpha)^{1 / 3}\right]^{2}} \\ 1-\alpha & (2 / 3) \alpha]-(1-\alpha)^{2 / 3} \\ (1-\alpha)^{2} & -\ln (1-\alpha)\end{array}$


nuclei of individual particles

$F_{3} \quad-\quad$ Random

nucleation with three nuclei of individual

$$
(1 / 2)(1-\alpha)^{3}
$$

$1 /(1-\alpha)$ particles

To determine the reaction mechanism, theoretical and the experimental curves must be compared. The theoretical curves can be obtained using Equation (8) and the $P(x)$ value is obtained by Equation (9).

$$
P(x)=\frac{e^{-x}}{x} \cdot \frac{x^{3}+18 x^{2}+86 x+96}{x^{4}+20 x^{3}+120 x^{2}+240 x+120}
$$

\subsection{Modified Coats-Redfern method}

The Modified Coats-Redfern method is also used to determine the activation energy and is defined by Equation (11).

$\ln \left[\frac{\beta}{T^{2}\left(1-\frac{2 R T}{E_{a}}\right)}\right]=\ln \left[-\frac{A R}{E_{a} \ln (1-\alpha)}\right]-\frac{E_{a}}{R T}$

Plotting $\ln \left(\beta / T^{2}\right)$ versus $1 / T$ and applying a linear fit the factor frequency (A) and the activation energy $\left(E_{a}\right)$ can be obtained. The slope gives $E_{a}$ and the intercept gives A.

\section{MATERIALS AND METHODS}

Commercial unsaturated polyester resin BP5788 and its green alternative from renewable resources were provided by Elekeiroz and used for thermal degradation studies.

Thermogravimetric analysis was used to determine the kinetic parameters. Samples (10 mg) were analysed in an SII Nanotechnology INC equipment, Exstar 6000 model, TG/DTA 6200 series, operating under nitrogen flow of $100 \mathrm{~mL} \cdot \mathrm{min}^{-1}$, in the $25-800^{\circ} \mathrm{C}$ temperature range.

\section{RESULTS AND DISCUSSIONS}

\subsection{Kinetics parameters}

Figure 1 illustrates all fitting using the four distinct kinetic models proposed. The obtained $\mathrm{R}^{2}$ values (from linear fitting) for all methods were higher than 0.88 for the traditional resin, and higher than 0.97 for the resin from renewable sources.

The activation energy $\left(E_{a}\right)$ values according to FWO, Friedman, and Coats-Redfern as function of the conversion degree are displayed Figure 2. The $E_{a}$ for Kissinger, also shows in Figure 1, not was included because this method use the peak of derivative of mass loss only, representing a limited conversion degree range.

According to the results of Figure 2, The activation energy seems to be dependent on the conversion degree $(\alpha=0.1-0.9)$ for both resins. This variation suggests that the degradation process of this polymer is complex, possibly with multiple reaction step mechanisms (parallel, consecutive, overlapping, reversible or their combination). This could be justified by the heterogeneity of the polymer nature [8]. 
For the traditional resin, the curves have similar shapes, and linear correlations between $E_{a}$ and $\alpha$ are found for $\alpha$ within $0.1-0.5$ and $0.7-0.9$. A maximum in $E_{a}$ was found around $\alpha=0.6$. And for the Friedman method, a more pronounced decrease occurred above $\alpha=0.7$. For the resin from renewable resources, a linear correlation between $E_{a}$ and $\alpha$ is found for $\alpha$ within $0.1-0.4$ and another one for $\alpha$ within $0.4-0.9$. And for Friedman method, an abrupt decrease in $E_{a}$ was observed when $\alpha$ goes from 0.4 from 0.5 and an abrupt increase was observed when $\alpha$ goes from 0.7 to 0.8 .

The difference in the trend in Figure 2 for Friedman method compared to the other two methods (FWO and modified Coats-Redfern) is justified considering that in the Friedman method, the derivative of the conversion $(d \alpha / d t)$ is used in the calculation, whereas in the others two methods the temperature is used.

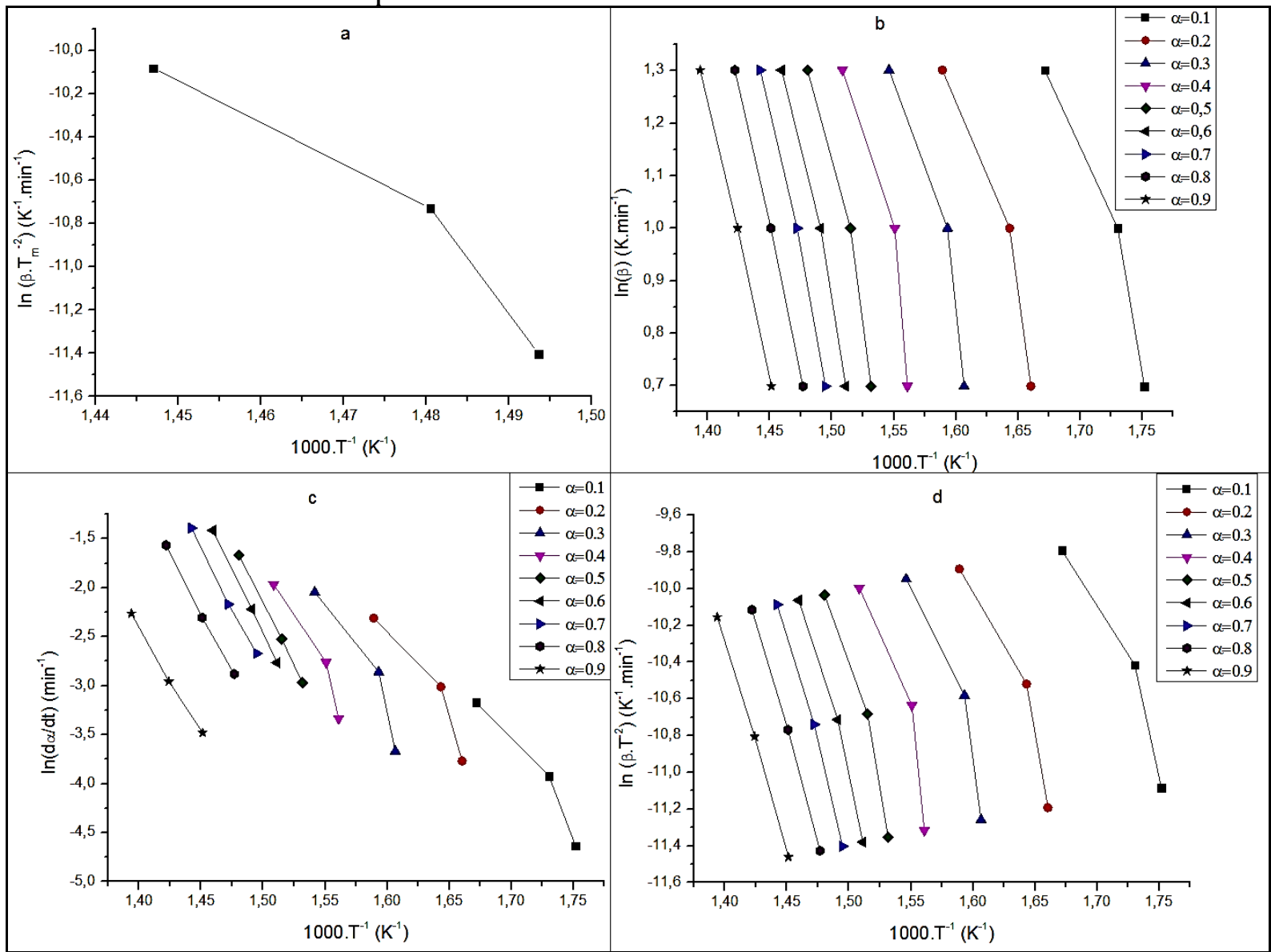

Figure 1 - Graphs used to determine the activation energy following Kissinger (a), FWO (b), Friedman (c) and Modified Coats-Redfern (d) methods. 


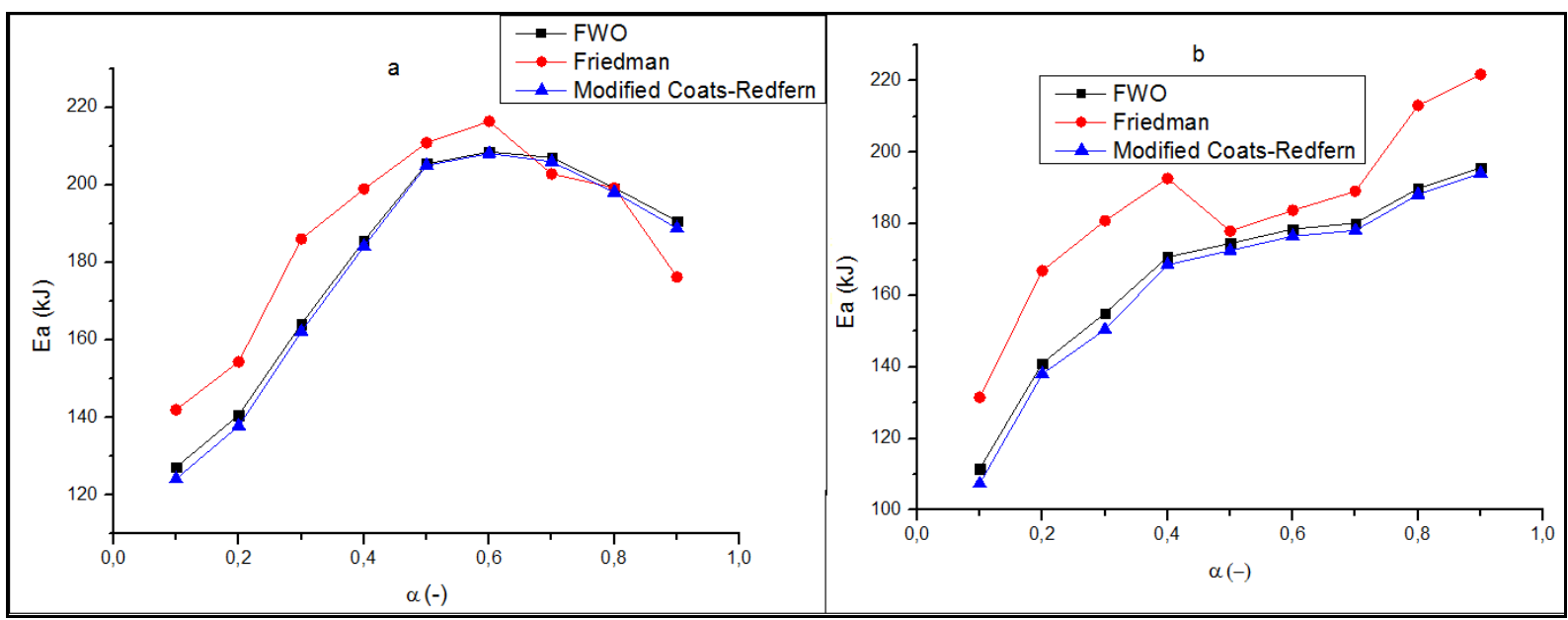

Figure 2 - The calculated activation energy as a function of the conversion degree for the traditional resin (a) and for the polyester from renewable resources (b).

By calculating the mean of all $E_{a}$ values from the four methods, $E_{I S O}$ was obtained. For the traditional resin was $189.79 \pm 37.82 \mathrm{~kJ}$, and the pre-exponential $(\ln (A))$ factor was $24.62 \pm$ $6.11 \mathrm{~min}^{-1}$. For the resin from renewable resources the values were $161.85 \pm 14.35 \mathrm{~kJ}$ and $18.42 \pm 2.36 \mathrm{~min}^{-1}$, respectively. And these values of $E_{\text {ISo }}$ were used to calculate the value of $z(\alpha)$ for the two resins.

\subsection{Degradation mechanisms}

Figure 3 illustrates the graphs used to determine the most probable the degradation mechanism. The most probable reaction degradation mechanism is compared considering the similarities in the curves. So, the resin can present different degradation mechanisms in different degrees.
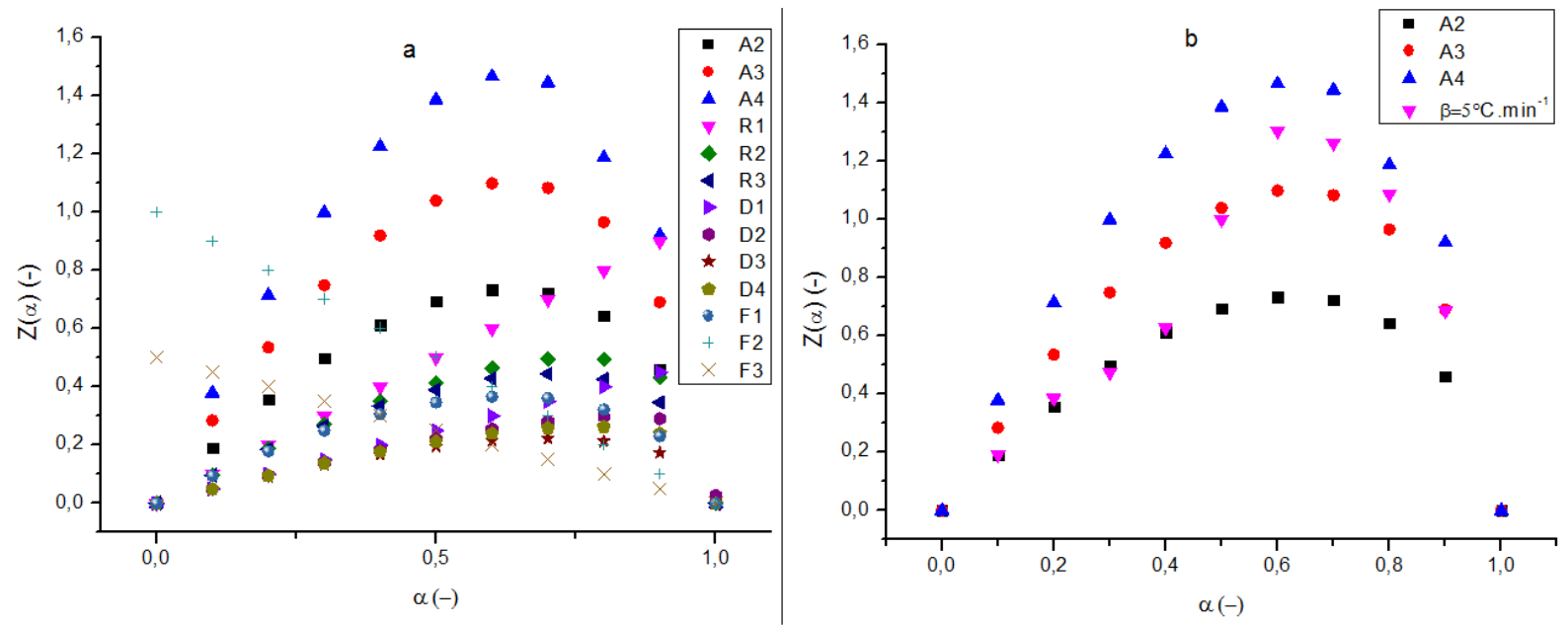

Figure 3 - Theoretical curves of the Criado degradation mechanism (a) and the graph obtained for the resin from renewable resource resin used in this work (b).

For the traditional resin, the shape of the curves did not follow a specific theoretical model for any heating rate, but $A$ type mechanism could be considered for the sample. In this case, all mechanisms were defined by proximity of $Z(\alpha)$. For $\alpha$ within $0.1-0.4$ (mechanism $A 2$ ), $\alpha=0.5$ (mechanism A3), $\alpha=0.6$ (mechanism A3), for $\alpha$ within $0.7-0.8$ (mechanism A4) and for $\alpha=0.9$ (mechanism A3). 
The same behaviour occurred for the resin from renewable resources, and, $A$ type mechanism could be considered. For $\alpha$ within $0.1-0.4$ (mechanism $A 2$ ), $\alpha=0.5$ (mechanism A3) and for $\alpha=0.9$ (mechanism A3). However, were cases that the mechanism was defined by the curve shape. It happened for $\alpha$ within $0.6-0.8$ (mechanism A4).

For the two resins, similarities in the mechanism from group $D(D 1, D 2, D 3, D 4)$, related to diffusion, from group $F(F 1, F 2, F 3)$, related to random nucleation, or from group $R(R 1$, $R 2, R 3)$, related to controlled reactions in the phase contour, could not be found.

Analysing all results, the overall difference between the two resins is small, i.e. the degradation mechanisms were similar. There were differences at some conversion values, e.g. the mechanism at $\alpha=0.6$ was $A 3$ for the traditional resin and $A 4$ for the other resin. Nevertheless, they were in the same group of mechanisms.

\section{CONCLUSIONS}

Analysing the activation energy results, the thermoset resins behaved as expected, and the $E_{a}$ values varied as a function of the conversion degree.

The degradation mechanisms for the traditional unsaturated polyester resin and for that from renewable resources were similar. Using the method described by Criado, the degradation mechanisms at different conversions were found to be of the group $A$ ( $A 2, A 3$ and $A 4$ ), suggesting a single group of degradation mechanisms, related to nucleation and growth, independently of the source of the resin. Also, by comparing different kinetic approaches, a more reliable estimate of the kinetic parameters can be obtained.

\section{REFERENCES}

[1] Ferreira, O. P., Alves, O. L., Macedo, J. D. S., Gimenez, I. D. F. and Barreto, L. S. (2007). Ecomateriais: desenvolvimento e aplicação de materiais porosos funcionais para proteção ambiental. Química Nova.

[2] Rühle, M., Dosch, H., Mittemeijer, E. J. and Van de Voorde, M. H. (Eds.). (2001). European White Book on Fundamental Research in Materials Science. Stuttgart: Max-Planck-Institut für Metallforschung.

[3] Badia, J. D., Santonja-Blasco, L., Moriana, R. and Ribes-Greus, A. (2010). Thermal analysis applied to the characterization of degradation in soil of polylactide: II. On the thermal stability and thermal decomposition kinetics. Polymer Degradation and Stability, 95(11), 2192-2199.

[4] Vyazovkin, S., Burnham, A. K., Criado, J. M., Pérez-Maqueda, L. A., Popescu, C. and Sbirrazzuoli, N. (2011). ICTAC Kinetics Committee recommendations for performing kinetic computations on thermal analysis data. Thermochimica acta, 520(1-2), 1-19.

[5] Criado, J. M., Malek, J. and Ortega, A. (1989). Applicability of the master plots in kinetic analysis of non-isothermal data. Thermochimica Acta, 147(2), 377-385.

[6] Paterson, W. L. (1971). Computation of the exponential trap population integral of glow curve theory. Journal of Computational Physics, 7(1), 187-190.

[7] Pérez-Maqueda, L. and Criado, J. (2000). The accuracy of Senum and Yang's approximations to the Arrhenius integral. Journal of thermal analysis and calorimetry, 60(3), 909-915.

[8] Poletto, M., Júnior, H. L. O. and Zattera, A. J. (2015). Thermal decomposition of natural fibers: kinetics and degradation mechanisms. Reactions and Mechanisms in Thermal Analysis of Advanced Materials, 515-545. 